This manuscript has been accepted for publication in:

Annals of the American Academy of Political and Social Science

(original submission 08/16/2021; final submission 11/13/2021)

\title{
The 'infodemic' infodemic: \\ Toward a more nuanced understanding of truth-claims and the need for (not) combatting misinformation
}

Nicole M. Krause, Isabelle Freiling, \& Dietram A. Scheufele

Nicole M. Krause is a doctoral candidate in the Department of Life Sciences Communication at the University of Wisconsin-Madison. Her work focuses on public perceptions of controversial science and issues of misinformation in science communication contexts.

Isabelle Freiling is a post-doctoral fellow in the Department of Communication at the University of Vienna. Her research focuses on media use and effects, as well as science and political communication, with a particular focus on social media and misinformation.

Dietram A. Scheufele is the Taylor-Bascom Chair in Science Communication and Vilas Distinguished Achievement Professor in the Department of Life Sciences Communication at the University of Wisconsin-Madison and the Morgridge Institute for Research. His work examines the social effects of emerging science and technology.

\section{Contact information:}

Krause: 331 Hiram Smith Hall, 1545 Observatory Drive, Madison, Wisconsin, USA, 53706; (608) 620-5212; nmkrause@wisc.edu.Freiling: isabelle.freiling@univie.ac.at. Scheufele: scheufele@wisc.edu. 


\begin{abstract}
(Mis)information scholarship struggles to assist policy actors in assessing the relative accuracy of science-related truth claims as well as their potential threat. This limited ability to produce translational research stems in part from conceptualizations of dis- and misinformation that pay insufficient attention to an information ecosystem in which defining "accuracy" is complicated by intersecting uncertainties associated with the nature of science, sociopolitical climates, and media systems. These uncertainties introduce compounding error in accuracy assessments, and they demand more nuanced understandings of mis- and disinformation than those which currently underlie discussions of the "infodemic." Here, we propose a framework for evaluating claims in terms of a collection of intersecting attributes beset by uncertainty. We apply our framework to real-world examples and conclude by discussing implications for research and action on (mis)information in a socio-scientific world where true and false claims are not as distinct as they are sometimes purported to be.
\end{abstract}

Keywords: misinformation, disinformation, truth claims, classification, conceptualization, science, risk 
Although scientists and policy actors have long attempted to categorize forms and attributes of mis- and disinformation (Soprano et al. 2021), this work is difficult to integrate. Classifications variously center on (combinations of) intent (Freelon and Wells 2020), facticity (Tandoc, Lim, and Ling 2017), authenticity (Glasser, Varma, and Zou 2018), falsifiability (Berinsky 2017), and other factors, often with the goal of typologizing claims as misinformation, disinformation, pseudoscience, conspiracy, rumor, native advertising, and so on (for an overview, see Scheufele and Krause 2019). As this work proliferates, the constellation of concepts drifts from a unifying logic. For example, a criterion like "intent" (Wardle 2017) is not obviously relevant to classifying claim accuracy, though it may be a useful criterion for assessing relative threat, albeit a blunt one. Meanwhile, others have argued that "some types of misinformation hold more consequence for public health behavior and outcomes than others" (Southwell et al. 2019, 282), thus highlighting variability in the relative threat claims might pose.

This lack of clear attention to the need to assess both claim accuracy and relative threat simultaneously in research and action on misinformation contributes to disjointed academic insights (Jerit and Zhao 2020), creates difficulty applying research in practice, and leaves us unable to judge whether emerging meta-analyses are treating as interchangeable studies of "misinformation" that are conceptually distinct (e.g., Walter and Tukachinsky 2019). "Misinformation" is also becoming a near-meaningless catch-all term for "studies focused on the proliferation and impacts of false information" (Cacciatore 2021, 1), with many studies (including some of our own) utilizing oversimplified operationalizations, or offering no definition of misinformation whatsoever (for examples, see Vraga and Bode 2021, Freiling, Krause, Scheufele, et al. 2021, Nyhan et al. 2019). 
Overall, in the academic realm, we risk spawning an industry of solution-focused work which assumes that "false" science-related claims are easily identifiable—a flawed assumption which has already led to studies "correcting" false beliefs with misleading claims (e.g., Bode, Vraga, and Tully 2021). Although the limitations we highlight also apply to aggregate-level work on the scope of misinformation (e.g., Grinberg et al. 2019), our points are geared more urgently toward research on individual-level interventions, where the need for nuance is especially important. Without precision, we waste time and energy—or, worse, we may do collateral damage (Scheufele et al. 2021).

In the policy realm, fuzzy conceptualizations leave actors unable to discern the relative threat a claim might pose or the harm it might do to differentially vulnerable populations. Should we focus on inaccuracy, on characteristics of the source, or both? Interventions are also difficult to enact if the research that informs them asserts that the solution is effective only for claims that are not politically polarizing (e.g., Weeks 2018), when politically polarizing claims are precisely the types of claims that often concern policymakers and pose greater threats. When a framework for classifying truth claims cannot account for forms of sociopolitical distortion (e.g., elite salience) or science-related distortion (e.g., fluidity of evidence about COVID-19 masking) and the intersections between these distortions, the scientific community becomes an easy target for politically motivated attacks. A good example of this problem is so-called "Lancetgate," which occurred in summer 2020. When former President Trump touted the efficacy of hydroxychloroquine as a treatment for COVID-19, an outpouring of factual correctives appeared in the New York Times and elsewhere. These correctives to Trump's statements were based largely on scientific evidence published in the Lancet, which was subsequently retracted, quickly 
sparking accusations by right-wing outlets like Breitbart of political bias in the scientific community (Delingpole 2020).

Integrating existing work in ways that informs productive pathways for research and practice on misinformation therefore requires attention to the ecosystem in which (mis)information emerges and evolves, where assessing "accuracy" and "threat" is complicated by intersecting distortions arising from the nature of science, sociopolitical climates, and media systems. These distortions introduce error - and, therefore, uncertainty and variability - in the many criteria we use to assess accuracy and threat. While some existing frameworks (see above) do classify truth claims in terms of multiple, intersecting attributes (some of which are indeed conceived of as variable), none of them explicitly acknowledge that intersecting attributes can compound the possible error associated with our judgments in ways that influence the costs of intervention. In this essay, we argue that evaluation of claims as misleading and worthy (or not) of intervention should be based on (a) a set of attributes understood as variable on spectra rather than as simply dichotomous, (b) the relevance of any given attribute to a particular claim, and (c) the intersectionality of relevant attributes, including as compounding sources of error.

\section{Intersecting attributes of information: An integrated framework for evaluating truth claims}

Scientific truth claims are rarely clear-cut. Even laws like gravity are explanatory models that cannot technically be "proven." So why are we not concerned about fringe publics clinging to misguided beliefs that are at odds with scientific consensus on this topic? The answer is twofold: Frist, the topic of gravity is not surrounded by sociopolitical debate that would introduce additional uncertainties or distortions to scientific truth claims. Second, and related, false claims about gravity are unlikely to gain significant traction in legacy media or on online platforms. 
This example highlights three broad categories of uncertainty and distortion that can surround scientific truth claims, and which form the organizational structure of our framework: (1) scientific; (2) sociopolitical; and (3) distributional uncertainties. As we will show below, each of these three types of uncertainty or sources of error will dictate the classification of a scientific truth claim as more or less accurate and more or less threatening.

Within each category of uncertainty are specific claim attributes, each of which, as we explain below, is itself surrounded by variability, either across issues or over time. Our list of attributes is inspired in part by a long legacy of work in the risk and decision sciences demonstrating the need to understand any risk - including the risk posed by dubious claims (Krause et al. 2020) — not only in terms of factors related to uncertainty, but also in terms of societal relevance, the form and magnitude of possible harm, and the liability of social amplification or distortion (Krimsky and Golding 1992). By evaluating truth claims in terms of the degree to which any of these attributes is relevant on its own but also when intersecting with other attributes, researchers and policy actors can assess a claim's accuracy and potential threat simultaneously, as is necessary when deciding how (and whether) to mitigate (mis)information.

[Insert Table 1 about here.]

Attainability of knowledge. Some claims will face epistemological limitations that necessarily introduce uncertainty - e.g., methodological limitations make it difficult to define a kilogram (Freiling, Krause, Chen, et al. 2021), and earthquake forecasting is plagued by high error rates. A more extreme version of this attribute, however, appears in attempts to debunk unlikely and explosive claims, such as the COVID-19 virus escaping a research lab in China (Kessler 2021). Key information about this claim may never become available, or it may be lost. 
Fluidity of evidence. To philosophers of science, the fluidity of scientific "truth" is wellknown-it is the deliberate and self-correcting nature of science (Hansson 2021). However, the uncertainties associated with self-correcting science are intensified during fast-moving crises like COVID-19, during which science and policy face the impossible task of countering claims they know are wrong with science that might not turn out to be right, either (Scheufele et al. 2020).

Stability across policy contexts. A claim can be accurate in one policy context but misleading in another. Standardized testing for college is a useful example. Discussions about predictive or criterion-related validity of standardized instruments like the GRE or MCAT have long questioned the ability of these instruments to accurately predict future academic success. Instead, many argue, these instruments tap candidates' social class, socioeconomic background, and other sociodemographic confounds rather than an ability to succeed in different educational settings. The GRE, therefore, might be an accurate and reliable tool for assessing structural inequities, but it might inspire misleading claims if it is used to predict students' real-world success in graduate school. Does this mean that standardized tests were not able to offer any valid information about applicants and we mostly admitted the wrong students? No. Does it mean that standardized tests were used without a full appreciation of the distortions and uncertainties surrounding their claims? Yes.

Fallibility of experts. Defining accurate claims (in part) as those aligned with the best available science at the time they are made (e.g., Southwell et al., this issue) can often be useful but will fail when science is simply wrong. In the 2010s, a paper widely hyped by NASA (2010) triggered over a year of global news coverage before being debunked by other scientists. While this could be construed as the self-correcting nature of science, it also demonstrates why intersectionality matters for assessing truth claims - -i.e., sources of error originating in the 
scientific community can be amplified by intersecting sociopolitical influences. As West and Bergstrom $(2021,1)$ noted: "Most analyses of misinformation focus on popular and social media, but the scientific enterprise faces a parallel set of problems-from hype and hyperbole to publication bias and citation misdirection, predatory publishing, and filter bubbles." A more detailed discussion of these points and the ways that "misinformation creeps into science" are available elsewhere in this issue (see Swire-Thompson and Lazer).

Consistency of evaluation standards. Whose standards of accuracy evaluation do we use, and when? Early in the 2010s, the results of a single study—described as "an intellectual foundation" in support of austerity politics — shaped the Obama administration's policy to curb the domestic deficit, as well as Germany's austerity push for the Eurozone (Herndon, Ash, and Pollin 2014, 259). Yet, attempts to replicate it revealed errors and omissions that undermined the evidence for these policies (Herndon, Ash, and Pollin 2014). The original authors claimed the findings were valid and attributed the "kerfuffle," as they diminutively called it, to differential evaluation standards, arguing that "politicians, commentators and activists" had "exaggerated or misrepresented" their results (Reinhart and Rogoff 2013).

Acceptability of source error. President Trump made 30,573 dubious claims in almost four years in office (Kessler et al. 2021). Meanwhile, the New York Times posts multiple daily corrections. This comparison is flawed, but it raises the question: What error rates or timeliness of correction are adequate for claims to be "honest mistakes" versus "misinformation?" This question is instructive for politicians, but it is even more relevant for sources of scientific claims. A 2021 National Academy of Medicine report, for instance, defined credible sources as "offering information that is consistent with the best scientific evidence available at the time and employing processes to reduce conflict of interest and promote transparency and accountability" 
(Kington et al. 2021, 6-7). But what error rates are acceptable for a "credible source" to (not) be aligned with the best available scientific evidence? How many retractions or non-replications are acceptable for a peer-reviewed journal to remain a broker of credible science?

Associated harm. The potential harm associated with false belief in some claims will be much greater than it is for others. Misinformation might be considered particularly dangerous and worthy of interventions if it demonstrably influences attitudes or behaviors in ways that cause individual, societal, or institutional harm. For example, the individual and societal consequences of believing that the Earth is flat arguably situate this claim much lower on the associated harm spectrum than a claim such as "childhood vaccinations cause autism," which can lead both to individual death and to the reactivation of a previously eradicated disease.

(Elite) salience. Decades of work on news values (Galtung and Ruge 1965) and agendabuilding (Cobb and Elder 1971) shows that elite endorsements of claims increase their reach. Similarly, "influencers" in social networks have become the truth-defying equivalent of the "two-step flow" when they perpetuate misinformation (Katz and Lazarsfeld 1955). When it comes to identifying claims that warrant attention from academic or policy communities, elite endorsements matter. They are what move claims out of obscurity and into mainstream debate.

Algorithmic traction. Truth claims of the future will circulate via media that are capable of mutating, as algorithm-based dissemination platforms powered by artificial intelligence (AI) come to know us better than we know ourselves (Youyou, Kosinski, and Stillwell 2015). Companies that own these algorithms are incentivized to exploit human biases, to predictively model behavior, and to curate our content with unprecedented precision (Cacciatore, Scheufele, and Iyengar 2016). When we design machines to outperform humans, we should not be surprised when they do, and we should recognize that our efforts to arm humans against nefarious forms of 
content curation may be limited in the short-term, possibly futile in the long-term, or even used against us by systems that are designed to anticipate and respond to human behavior. Further, we should expect these platforms to evolve in ways we do not understand, either because we lack access to proprietary data, or because the AI has advanced beyond knowledge of its activities.

\section{Intersecting attributes: Using the framework}

Below, we use our framework to briefly trace three real-world science-related claims:

Claim 1: "Mask-wearing can safely mitigate the spread of COVID-19." The science of mask-wearing amidst COVID-19 was highly variable and contested. This fluidity of evidence made accuracy evaluation difficult, and these difficulties were amplified by both elite salience and different standards of evaluation among science and policy actors. Meanwhile, the associated harm linked to misperception on this topic was high. Uncertainty about the science of mask-wearing interacted in this case with associated harm and other forms of sociopolitical distortion to produce a combustive mix that demanded attention.

Claim 2: "Injecting bleach can help cure COVID-19." The fluidity of evidence for this claim is virtually zero, but several other intersecting attributes have been more variable. This claim's algorithmic traction was extremely high, partly because of its nonsensical novelty and partly because of excitement among liberal elites (elite salience) to catch former President Trump flat-footed, given that the "injecting bleach" claim was often falsely attributed to him (McGraw and Stein 2021), including by then-candidate Biden on the campaign trail (Lybrand 2020). A claim that should have been written off as press conference nonsense with little impact was catapulted into a false statement by Biden due to intersecting distortions that fed each other over time. 
Claim 3: "The Earth is flat." Belief that the Earth is flat has attracted renewed scholarly attention. As with the injecting bleach example, the fluidity of evidence is extremely low. Still, scholars are calling on "scientists and science communicators ... to engage [flat earther] communities where they are" (Landrum, Olshansky, and Richards 2019, 160). Our framework clarifies why this intuition to act might be misguided: elite salience and algorithmic traction are low, so the claim is less likely to become widely-repeated or endorsed in a manner that distorts its perceived accuracy. Beyond this, the associated harm is arguably quite low as well.

\section{Nuance matters ... for research and action on misinformation}

We have argued that existing research on misinformation insufficiently accounts for intersecting sources of variation in judgments of claim "accuracy," and, further, that it does not easily facilitate the simultaneous assessment of accuracy and potential threat, opening a gulf between research and practice. Many claims languish in a "gray area," conceptually and operationally. Although some may argue that we can still intervene in more black-and-white claims, we neglect improving our understanding and handling of gray claims at our peril, especially if correcting them can have unintended consequences. (See the "Lancetgate" example we mentioned earlier, where politically-salient fact-checks that relied on a shifting scientific evidence base resulted in accusations of bias against the scientific community.) In addition to providing a blueprint for evaluating the relative accuracy and potential harm posed by a given scientific truth claim, our framework therefore also has implications for the trajectory and shape of research and action on misinformation.

Researchers can do more coherent and policy-relevant work. Researchers who want to explain how (mis)information works or investigate interventions can use our framework to more systematically account for the complexity of claims and can operationalize them in more 
nuanced ways in study designs. Researchers will also be better able to compare results across studies and to connect bodies of literature — both theoretical and empirical — as addressing similar kinds of claims. For example, studies presenting empirical evidence about the efficacy of correctives to false claims that lack elite salience or algorithmic traction can situate themselves as distinct from studies attempting to correct claims where such attributes are relevant. Further, this framework helps researchers to admit that we cannot (and typically do not) focus on all attributes at once. Specifying which attributes form the focus of a given study (and which are left out by necessity) will help us assess, for instance, which controls to include in analyses. We can then talk with more clarity about our results, so they can better inform policy.

\section{Interventions can better account for sources of distortion. Current interventions to} mitigate misinformation are fraught with landmines. Fact-checking and accuracy prompts, for example, are not only sometimes agnostic to the scientific, sociopolitical, and distributional uncertainties that contextualize claims and form the basis of our framework, but they sometimes also assume that problems would be solved if only people knew the truth. There is, however, mounting evidence contradicting this "knowledge deficit model," as well as other alleged public "deficits" that become rallying points for seeming "crises" of public opinion about science (Krause et al. 2021). Therefore, while the urge to "do something" about false claims is understandable, we need to think carefully about how to best use our resources.

A good starting point for refining intervention research is to ask which kinds of truth claims are indeed dangerous, as enabled by our framework. Recognizing both the complexity of claims and the variation in threat they might pose should direct efforts toward the most problematic claims and ensure that those efforts are tailored to the factors that make the claim threatening. For example, if algorithmic traction is a key threat-maximizing factor for many 
claims, then we should design interventions accordingly. We might deploy algorithms to flag claims that accelerate through new media, or intentionally optimize correctives for algorithmic prioritization. Of course, as long as companies make money by privileging claims that resonate with pre-existing beliefs instead of promoting accuracy, this is a futile argument.

We can (and should) avoid creating an infodemic about the infodemic. The popular assertion that we are facing a novel misinformation crisis is an empirical claim, the veracity of which would require that misinformation has either (a) not existed before, (b) not existed at this scale, (c) not existed in its current form, or (d) not influenced behaviors in ways that are as harmful as what we see now. In all cases, claiming that the threat is "new" necessitates a multipoint time comparison and evidence of a delta, as well as reason to believe that the measurement of "misinformation" at each point is comparable.

Yet, as argued above, scholars of misinformation have had limited success in integrating and refining our conceptualizations to create clear "standards" for operationalization, which means that such analyses would be difficult, or at least beset by significant error. It is reasonable to suggest, then, that claims about the novelty, nature, or scope of misinformation problemsincluding an "infodemic" about COVID-19, or other topics—should be understood as carrying more uncertainty than some researchers are perhaps willing to admit. Limitations in how we understand what misinformation is (and wide variation in how we define it, if we define it at all) necessarily limits how confidently we can claim that an "infodemic" exists, what its contours are, and how problematic it is. 


\section{References}

Berinsky, Adam J. 2017. Rumors and health care reform: experiments in political misinformation. British Journal of Political Science 47 (2):241-262.

Bode, Leticia, Emily K. Vraga, and Melissa Tully. 2021. Correcting misperceptions about Genetically Modified Food on social media: Examining the impact of experts, social media heuristics, and the gateway belief model. Science Communication 43 (2):225-251.

Cacciatore, Michael A. 2021. Misinformation and public opinion of science and health: Approaches, findings, and future directions. Proceedings of the National Academy of Sciences 118 (15).

Cacciatore, Michael A., Dietram A. Scheufele, and Shanto Iyengar. 2016. The end of framing as we know it ... and the future of media effects. Mass Communication and Society 19 (1):7-23.

Cobb, Roger W., and C. Elder. 1971. The politics of agenda-building: An alternative perspective for modern democratic theory. Journal of Politics 33:892-915.

Delingpole, James. 7 June 2020. Delingpole: Lancetgate is a humiliation for Trump's medical critics. Breitbart. Available from www.breitbart.com.

Freelon, Deen, and Chris Wells. 2020. Disinformation as political communication. Political Communication 37 (2):145-156.

Freiling, Isabelle, Nicole M. Krause, Kaiping Chen, and Dietram A. Scheufele. 2021. The science of open (communication) science: Toward an evidence-driven understanding of quality criteria in communication research. Journal of Communication.

Freiling, Isabelle, Nicole M. Krause, Dietram A. Scheufele, and Dominique Brossard. 2021. Believing and sharing misinformation, fact-checks, and accurate information on social media: The role of anxiety during COVID-19. New Media \& Society.

Galtung, Johan, and Mari Holmboe Ruge. 1965. The structure of foreign news. Journal of Peace Research 2 (1):64-91.

Glasser, Theodore L., Anita Varma, and Sheng Zou. 2018. Native advertising and the cultivation of counterfeit news. Journalism 20 (1):150-153.

Grinberg, Nir, Kenneth Joseph, Lisa Friedland, Briony Swire-Thompson, and David Lazer. 2019. Fake news on Twitter during the 2016 U.S. presidential election. Science 363:374-378.

Hansson, Sven Ove. 20 May 2021. Science and pseudo-science. The Metaphysics Research Lab. Available from www.plato.stanford.edu. 
Herndon, Thomas, Michael Ash, and Robert Pollin. 2014. Does high public debt consistently stifle economic growth? A critique of Reinhart and Rogoff. Cambridge Journal of Economics 38 (2):257-279.

Jerit, Jennifer, and Yangzi Zhao. 2020. Political misinformation. Annual Review of Political Science 23 (1):77-94.

Katz, Elihu, and Paul F. Lazarsfeld. 1955. Personal influence. New York: The Free Press.

Kessler, Glenn. 25 May 2021. Fact check: How the Wuhan lab-leak theory for pandemic origin suddenly became credible. The Seattle Times. Available from www.seattletimes.com.

Kessler, Glenn, Salvador Rizzo, and Meg Kelly. 24 January 2021. Trump's false or misleading claims total 30,573 over 4 years. The Washington Post. Available from www.washingtonpost.com.

Kington, Raynard S. , Stacey Arnesen, Wen-Ying Sylvia Chou, Susan J. Curry, David Lazer, and Antonia M. Villarruel. 2021. Identifying credible sources of health information in social media: Principles and attributes, NAM perspectives. Washington, DC: National Academy of Medicine.

Krause, Nicole M., Isabelle Freiling, Becca Beets, and Dominique Brossard. 2020. Fact-checking as risk communication: the multi-layered risk of misinformation in times of COVID-19. Journal of Risk Research 23 (7-8):1052-1059.

Krause, Nicole M., Dietram A. Scheufele, Isabelle Freiling, and Dominique Brossard. 2021. The trust fallacy: Scientists' search for public pathologies is unhealthy, unhelpful, and ultimately unscientific. American Scientist 109 (4):226-231.

Krimsky, Sheldon, and Dominic Golding. 1992. Social theories of risk. Westport, CT: Praeger.

Landrum, Asheley R., Alex Olshansky, and Othello Richards. 2019. Differential susceptibility to misleading flat earth arguments on youtube. Media Psychology 24 (1):136-165.

Lybrand, Holmes. 15 October 2020. Fact check: Biden's comments on Trump and bleach. CNN. Available from www.edition.cnn.com.

McGraw, Meridith, and Sam Stein. 23 April 2021. It's been exactly one year since Trump suggested injecting bleach. We've never been the same. Politico. Available from www.politico.com.

NASA. 29 November 2010. NASA Sets News Conference on Astrobiology Discovery. Available from www.nasa.gov.

Nyhan, Brendan, Ethan Porter, Jason Reifler, and Thomas J. Wood. 2019. Taking fact-checks literally but not seriously? The effects of journalistic fact-checking on factual beliefs and candidate favorability. Political Behavior 42 (3):939-960. 
Reinhart, Carmen M., and Kenneth S. Rogoff. 25 April 2013. Debt, growth and the austerity debate. The New York Times. Available from www.nytimes.com.

Scheufele, Dietram A., and Nicole M. Krause. 2019. Science audiences, misinformation, and fake news. Proceedings of the National Academy of Sciences 116 (16):7662-7669.

Scheufele, Dietram A., Nicole M. Krause, and Isabelle Freiling. 2021. Misinformed about the "infodemic"? Science's ongoing struggle with misinformation. Journal of Applied Research in Memory and Cognition 10 (4): 522-526.

Scheufele, Dietram A., Nicole M. Krause, Isabelle Freiling, and Dominique Brossard. 2020. How not to lose the COVID-19 communication war. Issues in Science and Technology.

Soprano, Michael, Kevin Roitero, David La Barbera, Davide Ceolin, Damiano Spina, Stefano Mizzarro, and Gianluca Demartini. 2021. The many dimensions of truthfulness: Crowdsourcing misinformation assessments on a multidimensional scale. Information Processing \& Management 58 (6):102710.

Southwell, Brian G., Jeff Niederdeppe, Joseph N. Cappella, Anna Gaysynsky, Dannielle E. Kelley, April Oh, Emily B. Peterson, and Wen-Ying Sylvia Chou. 2019. Misinformation as a misunderstood challenge to public health. American Journal of Preventive Medicine 57 (2):282-285.

Tandoc, Edson C., Zheng Wei Lim, and Richard Ling. 2017. Defining "fake news". Digital Journalism 6 (2):137-153.

Vraga, Emily K., and Leticia Bode. 2021. Addressing COVID-19 Misinformation on Social Media Preemptively and Responsively. Emerging Infectious Diseases 27 (2):396-403.

Walter, Nathan, and Riva Tukachinsky. 2019. A meta-analytic examination of the continued influence of misinformation in the face of correction: How powerful is it, why does it happen, and how to stop it? Communication Research 47 (2):155-177.

Wardle, Claire. 2017. Fake news. It's complicated. First Draft Footnotes. Available from www.medium.com.

Weeks, Brian E. 2018. Media and political misperceptions. In Misinformaton and Mass Audiences, eds. Brian G. Southwell, Emily A. Thoson, and Laura Shelbe, 140-156. Austin, Texas: University of Texas Press.

West, Jevin D., and Carl T. Bergstrom. 2021. Misinformation in and about science. Proceedings of the National Academy of Sciences 118 (15):e1912444117.

Youyou, Wu, Michal Kosinski, and David Stillwell. 2015. Computer-based personality judgments are more accurate than those made by humans. Proceedings of the National Academy of Sciences of the United States of America 112 (4):1036-1040. 
Table 1. Intersecting attributes introducing variability in evaluation of claim accuracy and threat.

\begin{tabular}{|c|c|c|}
\hline Uncertainty type & Attribute & Key question(s) \\
\hline \multirow[t]{4}{*}{ Scientific } & $\begin{array}{l}\text { Attainability of } \\
\text { knowledge }\end{array}$ & $\begin{array}{l}\text { What obstacles prevent attaining "certainty?" Are some } \\
\text { claims plagued by more irremovable uncertainty than } \\
\text { others? }\end{array}$ \\
\hline & $\begin{array}{l}\text { Fluidity of } \\
\text { evidence }\end{array}$ & $\begin{array}{l}\text { How settled is the science? How do over-time changes } \\
\text { in certainty of the fact base undermine our ability to } \\
\text { diagnose misinformation? }\end{array}$ \\
\hline & $\begin{array}{l}\text { Stability across } \\
\text { policy contexts }\end{array}$ & $\begin{array}{l}\text { How much real-world predictive validity does a claim } \\
\text { have? Can available science provide accurate answers } \\
\text { to relevant social or political questions? }\end{array}$ \\
\hline & $\begin{array}{l}\text { Fallibility of } \\
\text { experts }\end{array}$ & $\begin{array}{l}\text { What is the evidence base to falsify expert claims? How } \\
\text { can we inform policy with corrective interventions? Is } \\
\text { this claim (likely to be) hyped? }\end{array}$ \\
\hline \multirow[t]{3}{*}{ Sociopolitical } & $\begin{array}{l}\text { Consistency of } \\
\text { evaluation } \\
\text { standards }\end{array}$ & $\begin{array}{l}\text { Do policy makers evaluate this claim differently from } \\
\text { academics? How can we deal with false claims that are } \\
\text { dismissed as "sarcasm" or "par for the course" for } \\
\text { political actors? }\end{array}$ \\
\hline & $\begin{array}{l}\text { Acceptability } \\
\text { of source error }\end{array}$ & $\begin{array}{l}\text { What rates of error are expected for different sources? } \\
\text { When do errors become misinformation? What error } \\
\text { rate is acceptable in peer-refereed scientific } \\
\text { publications, preprints, science journalism, etc.? }\end{array}$ \\
\hline & $\begin{array}{l}\text { Associated } \\
\text { harm }\end{array}$ & $\begin{array}{l}\text { To what extent will misperceptions on the topic raise } \\
\text { the potential for catastrophe, ruin, physical harm, or } \\
\text { other forms of damage, at the individual, societal, or } \\
\text { institutional levels? }\end{array}$ \\
\hline \multirow[t]{2}{*}{ Distributional } & (Elite) salience & $\begin{array}{l}\text { Does the claim have large-scale societal relevance vs. } \\
\text { fringe appeal? Are elite endorsements of the claim } \\
\text { likely? }\end{array}$ \\
\hline & $\begin{array}{l}\text { Algorithmic } \\
\text { traction }\end{array}$ & $\begin{array}{l}\text { Will corrections give digital oxygen to this claim? Is } \\
\text { there an "if it bleeds it leads" equivalent for outrageous } \\
\text { falsehoods for platforms that fiscally thrive on user } \\
\text { engagement? }\end{array}$ \\
\hline
\end{tabular}

